\title{
Evolution of electron temperature in inductively coupled plasma
}

Hyo-Chang Lee, B. H. Seo, Deuk-Chul Kwon, J. H. Kim, D. J. Seong, S. J. Oh, C.-W. Chung, K. H. You, and ChaeHo Shin

Citation: Appl. Phys. Lett. 110, 014106 (2017); doi: 10.1063/1.4971980

View online: http://dx.doi.org/10.1063/1.4971980

View Table of Contents: http://aip.scitation.org/toc/apl/110/1

Published by the American Institute of Physics 


\title{
Evolution of electron temperature in inductively coupled plasma
}

\author{
Hyo-Chang Lee, ${ }^{1, \text { a) }}$ B. H. Seo, ${ }^{2}$ Deuk-Chul Kwon, ${ }^{3}$ J. H. Kim, ${ }^{1, a)}$ D. J. Seong, ${ }^{1}$ S. J. Oh, ${ }^{4}$ \\ C.-W. Chung, ${ }^{4}$ K. H. You, ${ }^{1}$ and ChaeHo Shin ${ }^{5}$ \\ ${ }^{1}$ Center for Vacuum Technology, Korea Research Institute of Standard and Science, Daejeon 305-340, \\ South Korea \\ ${ }^{2}$ Applied Physics, California Institute of Technology, Pasadena, California 91125, USA \\ ${ }^{3}$ Plasma Technology Research Center, Nation Fusion Research Institute, Gunsan 573-540, South Korea \\ ${ }^{4}$ Department of Electrical Engineering, Hanyang University, 17 Haengdang-dong, Seongdong-gu, \\ Seoul 133-791, South Korea \\ ${ }^{5}$ Division of Industrial Metrology, Korea Research Institute of Standard and Science, Daejeon 305-340, \\ South Korea
}

(Received 6 October 2016; accepted 27 November 2016; published online 6 January 2017)

It is generally recognized that the electron temperature $T_{e}$ either remains constant or decreases slightly with plasma power (plasma density). This trend can be simply verified using a single-step or multi-step fluid global model. In this work, however, we experimentally observed that $T_{e}$ evolved with plasma power in radio frequency (RF) inductively coupled plasmas. In this experiment, the measured electron energy distributions were nearly Maxwellian distribution. In the low RF power regime, $T_{e}$ decreased with increasing plasma power, while it increased with plasma power in the high RF power regime. This evolution of $T_{e}$ could be understood by considering the coupling effect between neutral gas heating and stepwise ionization. Measurement of gas temperature via laser Rayleigh scattering and calculation of $T_{e}$ using the kinetic model, considering both multi-step ionization and gas heating, were in good agreement with the measured value of $T_{e}$. This result shows that $T_{e}$ is in a stronger dependence on the plasma power. Published by AIP Publishing.

[http://dx.doi.org/10.1063/1.4971980]

Electron temperature $\left(T_{e}\right)$ is one of the most important plasma parameters in both industrial plasma processes and fundamental laboratory research. ${ }^{1-3}$ In industrial semiconductor, display, and solar-cell plasma processes, such as etching and deposition, key mechanism for achieving highquality device fabrication is the physical and chemical reactions. The ion energy/flux impinging on the wafer, which is affected by the $T_{e}$, determines the physical reaction. This is because, the sheath potential and the number density of ions change depending on the value of $T_{e}$. The radical density and its composition, which are related to the chemical reaction on the wafer surface, are predominantly governed by $T_{e}$ or electron energy distribution function (EEDF). ${ }^{4}$ In laboratory research, $T_{e}$ is a fundamental plasma parameter that aids in the understanding of the discharge characteristics ${ }^{5-10}$ and electron sustainment mechanisms, ${ }^{11-13}$ such as electron heating.

It was generally recognized that $T_{e}$ is decoupled ${ }^{1,14,15}$ or slightly coupled ${ }^{16,17}$ to the absorbed plasma power (or plasma density $n_{e}$ ) in inductively coupled plasmas (ICPs). In the single-step ionization global model based on fluid analysis, $T_{e}$ is determined by particle conservation, while $n_{e}$ is obtained from the power balance equation. Thus, $T_{e}$ remains unaltered with increasing plasma power or $n_{e}$. In the multi-step ionization global model, which uses modified fluid approximation, ${ }^{16} T_{e}$ is slightly decreased with plasma power or $n_{e}$, because multistep ionization caused by excited (mainly metastable) atoms results in a decrease in the collisional energy loss $\left(\varepsilon_{c}\right)$ per electron-ion pair created, although the rate of decrease of $T_{e}$ is

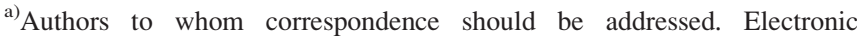
addresses: 1hc@kriss.re.kr and jhkim86@kriss.re.kr
}

significantly low compared to the variation in $n_{e}$. Recently, effect of the EEDF was also considered in the global model. ${ }^{18,19}$

In this study, however, we observed an abnormal variation in $T_{e}$ with the plasma power in ICP, which implies that the $T_{e}$ is strongly coupled to the plasma power and $n_{e}$. This variation in $T_{e}$ was analyzed via laser Rayleigh scattering measurements and the improved kinetic model considering both multi-step ionization and gas heating.

The experiment was investigated in a radio frequency (RF) ICP reactor (Fig. 1). The chamber had a cylindrical shape with an inner diameter of $26 \mathrm{~cm}$ and a height of $18 \mathrm{~cm}$ from top plate to bottom electrode. The sidewall and bottom electrode were made of stainless steel, and the top plate was made of ceramic. An RF power of $13.56 \mathrm{MHz}$ was applied to an antenna coil, and an automatically controlled L-type impedance matching network was placed between the RF power generator and the antenna coil to minimize the reflected power. A rotary vane pump and a turbo-molecular vacuum pump were used to maintain the base pressure below 5 $\times 10^{-6}$ Torr. Argon gas was used to sustain the plasma, and the gas flow rate was controlled by a mass flow controller.

To measure the plasma parameters, such as $T_{e}$ and the electron energy probability function (EEPF), an RFcompensated single Langmuir probe was placed at the center of the discharge region. A schematic of the Langmuir probe configuration can be seen in Refs. 19-21. Briefly describing, the probe had a tip made of tungsten wire with a diameter of $0.1 \mathrm{~mm}$ and a length of $6 \mathrm{~mm}$; the probe body was made of a ceramic tube with a telescoping structure. ${ }^{22}$ To compensate for the RF fluctuations in the measurement of the currentvoltage $(I-V)$ curve, a reference holder and resonance filters 
corresponding to a fundamental frequency of $13.56 \mathrm{MHz}$ and a second harmonic frequency of $27.12 \mathrm{MHz}$ were included in the Langmuir probe system. The EEPF is obtained by using the AC superposition method. ${ }^{23-27}$ This method can offer the reliable EEPF because a small error of the $I-V$ curve in the conventional method (direct second derivative method) can give enormous distortion in the EEPF. When a sinusoidal voltage $\left(\mathrm{v}_{0}\right)$ is applied to the measured $I-V$ curve, the plasma current can be obtained using Taylor series expansion, and the second harmonic current $I_{2 \omega}$ is given as $I_{2 \omega} \approx \frac{v_{0}^{2}}{4} \frac{d^{2} I(V)}{d V^{2}}$. Because $\frac{d^{2} I_{e}(V)}{d V^{2}} \gg \frac{d^{2} I_{i}(V)}{d V^{2}}$ except when the probe potential is very close to or larger than the plasma potential, ${ }^{23,24} I_{2 \omega}$ can be written as

$$
I_{2 \omega} \approx \frac{\mathrm{v}_{0}^{2}}{4} \frac{d^{2} I_{e}(V)}{d V^{2}} .
$$

Here, $I_{e}(V)$ and $I_{i}(V)$ are the electron current and the ion current. Because the EEDF $g_{e}(\varepsilon)$ is proportional to the second derivative of the electron current $I_{e}(V)$ in isotropic plasmas $^{1,28}$ as $g_{e}(\varepsilon)=\frac{2 m}{e^{2} A}\left(\frac{2 \varepsilon}{m}\right)^{0.5} \frac{d^{2} e_{e}(V)}{d V^{2}}$, the measurement of $I_{2 \omega}$ gives the value of EEDF or EEPF. Here, the EEPF $f_{e}(\varepsilon)$ is related to the $g_{e}(\varepsilon)$ as $g_{e}(\varepsilon)=\varepsilon^{0.5} f_{e}(\varepsilon) . T_{e}$ is obtained from the EEDF as follows:

$$
T_{e}=\frac{2}{3} \frac{1}{n_{e}} \int_{0}^{\infty} \varepsilon g_{e}(\varepsilon) d \varepsilon,
$$

where $n_{e}=\int_{0}^{\infty} g_{e}(\varepsilon) d \varepsilon$.

Figure 2 shows the measured $T_{e}$ and EEPF with increasing RF power at an argon gas pressure of 50 mTorr. As the RF power increases, a variation in the trend of $T_{e}$ was observed. $T_{e}$ decreased when the RF power increased from $100 \mathrm{~W}$ to $500 \mathrm{~W}$, at first. However, $T_{e}$ increased with a further increase in the RF power. This change in $T_{e}$ was also found in both discharge center and radial boundary by using the EEPF measurement and floating harmonic technique (not shown here), ${ }^{29}$ and the results were in identical trends to Fig. 2. The evolution in $T_{e}$ is interesting because it was well known that $T_{e}$ will either remain constant or slightly decrease with $n_{e}$ in the single-step or multi-step ionization global model. ${ }^{1,16}$ One possible explanation for this trend in $T_{e}$ may be the dramatic evolution of the EEPF through the electron heating effect ${ }^{11,21,30-37}$ or electronelectron collisions. ${ }^{17,26,38,39}$ However, our experiment was conducted in plasmas having nearly Maxwellian EEPFs. As shown in Fig. 2(b), the measured EEPFs are in the Maxwellian shape except for a slight different tail in the inelastic electron energy range. Therefore, the behavior of $T_{e}$ is not due to the evolution of the EEPF. In the measured EEPFs, the variation in $T_{e}$ could also be observed clearly through changes in the slope of the EEPF in elastic electron energy range, which is inversely proportional to $T_{e}$.

This evolution of $T_{e}$ can be understood by coupling effect between neutral gas heating and stepwise ionization. When stepwise ionization is considered in plasmas, the ionization efficiency is enhanced because the ionization energy of the excited atoms is lower than that of the grounded atoms. For example, electron energy of $11.5-11.7 \mathrm{eV}$ is required to excite ground state argon atoms to the metastable state; atoms in the metastable state have a long radiative lifetime compared to other excited state atoms. ${ }^{1}$ This means that an additional energy of only about $4-5 \mathrm{eV}$ is needed for the ionization process to initiate, which is much lower than the single collision ionization energy $(15.76 \mathrm{eV})$ of the ground state atoms. Because the stepwise ionization process is enhanced with increasing $n_{e}, \varepsilon_{c}$ is reduced with an increase in the RF power. Therefore, $T_{e}$ decreases with an increase in the plasma power in the stepwise ionization model.

However, this model did not account for the effect of the neutral gas heating. The neutral gas is heated by various collisions in the plasma. There are many possible gasheating mechanisms in noble gas plasmas. One mechanism is electron-neutral collisions. But, electron-neutral collisions may account for only a small fraction of gas heating because in such collisions, the energy transfer ratio is dependent on the mass ratio although $T_{e}$ is much higher than the gas temperature $T_{g}$. In the case of molecular gas plasmas, gas heating due to the energy released in dissociation processes can be significant. The other mechanism is ion-neutral collisions, such as momentum collisions and charge transfer collisions. In these cases, the energy transfer ratio will be very high owing to the similar masses involved although the ion temperature $T_{i}$ is not high. However, if ion acceleration by ambipolar potential or sheath potential is considered in ionneutral collisions, a sufficiently strong gas heating effect may occur; this effect becomes more prominent as $n_{e}$ increases.

The gas heating results in an inverse variation in $T_{e}$ compared to the variation of $T_{e}$ via the stepwise ionization because an increase in $T_{g}$ results in a decrease in the gas density in terms of pressure balance; this causes $T_{e}$ to increase with increasing $n_{e}$. To understand the variation in $T_{e}$ by this coupling effect between gas heating and stepwise ionization, measurement of $T_{g}$ using laser Rayleigh scattering and calculation of $T_{e}$ using the kinetic model considering both multi-step ionization and gas heating were performed.

Figure 3(a) shows the plot of the measured $T_{g}$ versus RF power obtained using the laser Rayleigh scattering measurement system shown in Fig. 1. The measurement procedure and method can be found in Refs. 40 and 41 . The apparatus is described as follows. A frequency-doubled Nd:YAG laser (Powerlite 9010, Continuum, Inc.) with a pulse energy of $300 \mathrm{~mJ}$ and a frequency of $10 \mathrm{~Hz}$ was used to measure the scattering signals. A laser beam, which is vertically polarized, was focused at the center of the chamber. The scattered signals were collected by the two lenses-the monochromator and the intensified charged coupled device (ICCD) camera (PIMAX, Princeton Instruments). The Rayleigh scattering signals of 2000 shots were accumulated for $200 \mathrm{~s}$. The laser energy was also monitored at the end of the laser path. From the measurements of the Rayleigh scattering signals with and without plasma, $T_{g}$ can be determined as follows:

$$
T_{g}=T_{\text {ref }} \frac{P_{\text {ref }}-P_{\text {stray }}}{P_{\text {plasma }}-P_{\text {stray }}} .
$$

Here, $T_{\text {ref }}, P_{\text {ref }}, P_{\text {plasma }}$, and $P_{\text {stray }}$ are the room temperature, the wavelength-integrated scattered power of the Rayleigh 


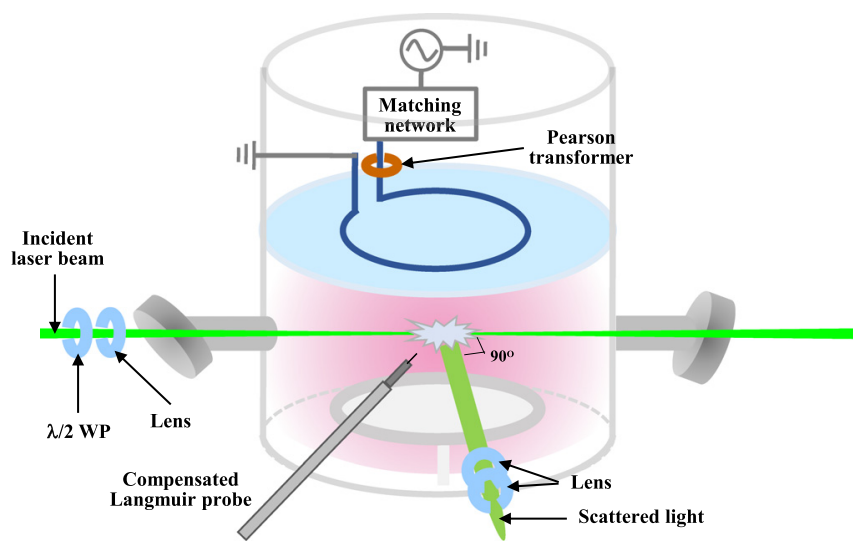

FIG. 1. Experimental setup of an inductively coupled plasma reactor.

signal without plasma as the reference measurement, the wavelength-integrated scattered power of the Rayleigh signal with plasma, and the wavelength integrated scattered power of stray light in a vacuum, respectively. The measured $T_{g}$ is indicated in Fig. 3(a). When the RF power of $100 \mathrm{~W}$ was applied, $T_{g}$ was approximately $300 \mathrm{~K}$. As the RF power increased from $100 \mathrm{~W}$ to $500 \mathrm{~W}, T_{g}$ increased slightly. When more RF power was applied, $T_{g}$ increased remarkably upto $946 \mathrm{~K}$. This trend in the variation of $T_{g}$ is in good agreement with other experiments. ${ }^{42-44}$

Figures 3(b) and 3(c) present the measured power absorption and $n_{e}$ against RF power. When the RF power increased from $100 \mathrm{~W}$ to $500 \mathrm{~W}, n_{e}$ increases (Fig. 3(c)) and $\mathrm{T}_{\mathrm{e}}$ decreases (Fig. 2(a)) due to enhanced power absorption and stepwise ionization. This increase in the $n_{e}$ results in the gas heating and thus, the gas temperature is increased (Fig. $3(\mathrm{a})$ ). When more RF power is applied, the $n_{e}$ reaches to a high plasma density regime as $5 \times 10^{11} \mathrm{~cm}^{-3}-9 \times 10^{11} \mathrm{~cm}^{-3}$ (Fig. 3(c)), which results in strong gas heating, as shown in Fig. 3(a). Even though the stepwise ionization process also occurs in the high power (high $n_{e}$ ) regime, the remarkable gas heating and high $n_{e}$ make the neutral density depleted. Therefore, the plasma production is saturated (Fig. 3(c)). It is noted that this behavior makes electron temperature to be increased (Fig. 2(a)) via transition of the discharge characteristics from being stepwise ionization-dominated to gas heating-dominated.

As shown in Figs. 2 and 3, the increase in $T_{g}$ can significantly affect plasma parameters, such as $T_{e}$ and $n_{e}$. To see this dynamics of the plasma parameters, we numerically solved the global transport equations and the Fokker-Planck equation. ${ }^{45-49}$ The global transport equation is given as

$$
\frac{\partial n_{i}}{\partial t}=\sum_{j} R_{g, j}+\frac{Q_{i}}{\Omega}-\sum_{j} R_{l, j}-n_{i}\left(\frac{V_{\text {pump }}}{\Omega}+\nu_{l}^{i}\right),
$$

where $n_{i}$ is the density of the species $i, Q_{i}$ is the gas flow rate of species $i, \Omega$ is the volume of the chamber, and $V_{\text {pump }}$ is the pumping speed. The loss speed of the ions at the plasmasheath boundary is assumed to be the Bohm velocity; thus, the loss frequency of the $i$ th ion species becomes $\nu_{l}^{i}=A_{\text {eff }}$ $\sqrt{T_{e} / M_{i}} / \Omega$. Here, $M_{i}$ is the ion mass and $A_{\text {eff }}$ is the effective surface area shown as
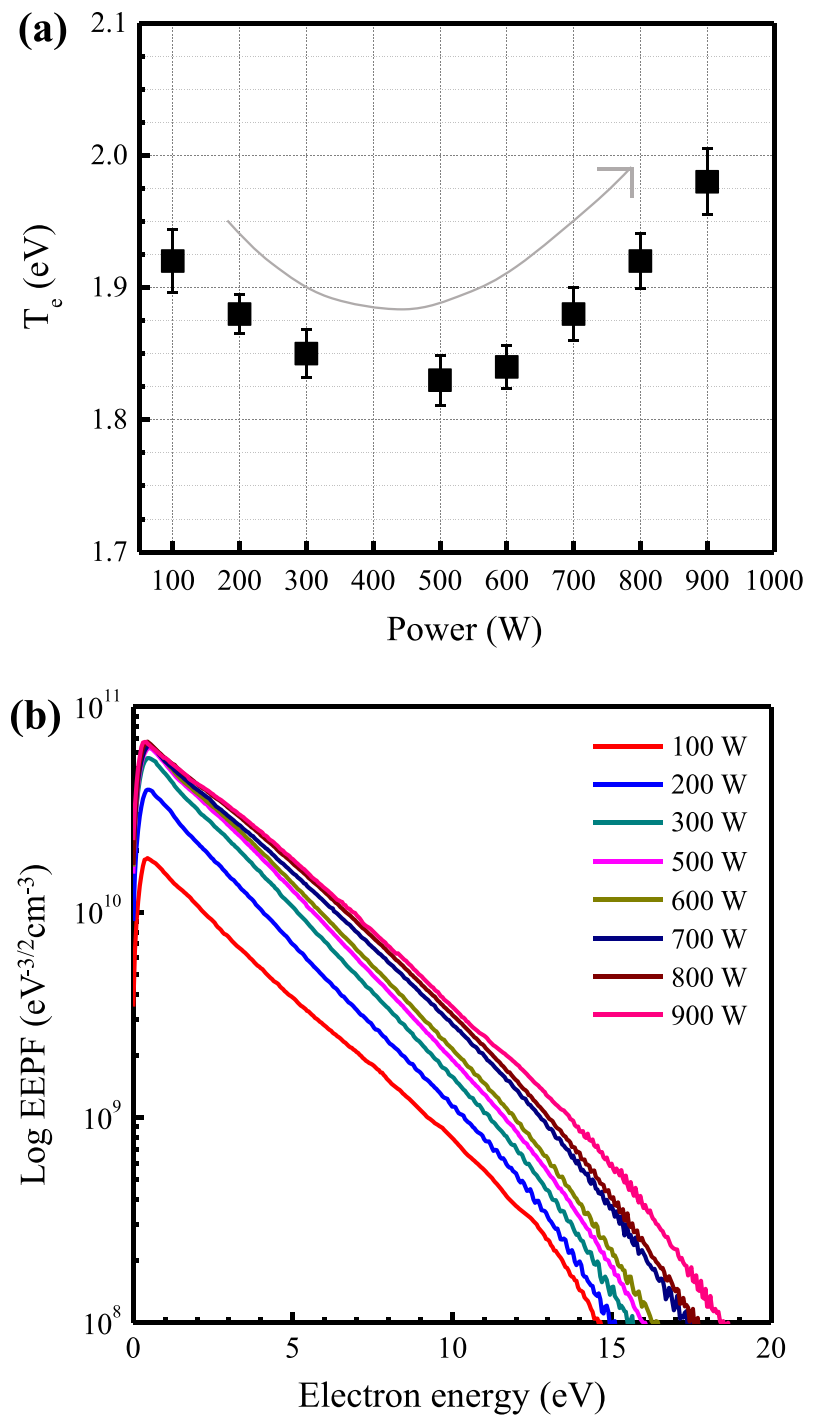

FIG. 2. Experimental results of (a) electron temperature $\left(\mathrm{T}_{\mathrm{e}}\right)$ and (b) electron energy probability function (EEPF) with RF power.

$$
A_{e f f}=\left.\frac{n_{i s}}{n_{i}}\right|_{\text {axial }} 2 \pi R_{p}^{2}+\left.\frac{n_{i s}}{n_{i}}\right|_{\text {radial }} 2 \pi R_{p} L_{p}
$$

where $R_{p}$ is the plasma radius, $L_{p}$ is the plasma length, and the ratio of sheath edge density $n_{i s}$ to the bulk average density $n_{i}$ is derived in Refs. 1 and 18. $R_{g, j}$ and $R_{l, j}$ are the reaction rates
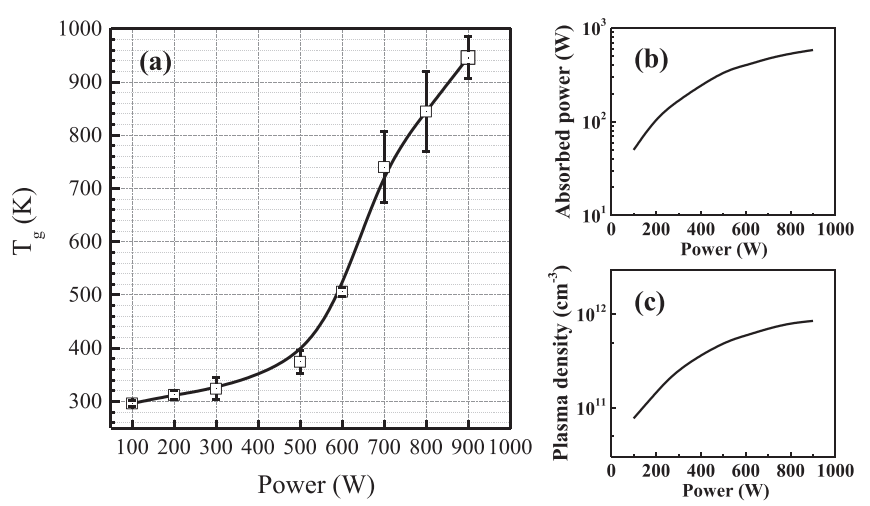

FIG. 3. (a) Measured gas temperature, (b) RF power absorption, and (c) plasma density with RF power. 
of the various generation and loss processes of the species $i$, respectively. In particular, the reaction rates for electronneutral collisions are calculated as the product of the reactants' densities and the rate coefficient $k$ of the reaction as shown in

$$
k=\int_{0}^{\infty} f_{0}(\varepsilon) \sigma(\varepsilon) v d \varepsilon
$$

Here, $\sigma(\varepsilon)$ is the electron-neutral collision cross sections. In this work, $4 \mathrm{~s}_{\mathrm{r}}, 4 \mathrm{~s}_{\mathrm{m}}$, and $4 \mathrm{p}$ excited states are considered and the reactions are given in Refs. 16 and 19, and radiation trapping $^{50,51}$ is not considered in the kinetic model.

It should be noted that our measurement shows Maxwellian EEPFs with depletion on the high-energy part, and the depleted part becomes replenished with RF powers due to the electron-electron collisions. Because the high energy tail on the EEPF can result in change of the stepwise ionization process with the enhanced excitation states, the EEDF should be considered in the model, and the EEDF is calculated from the Fokker-Planck equation as follows

$$
\frac{1}{v} \frac{\partial}{\partial \varepsilon} v\left[v\left(D_{\varepsilon}+D_{e e}+D_{e n}\right) \frac{\partial f_{0}}{\partial \varepsilon}+\left(V_{e e}+V_{e n}\right) f_{0}\right]=I
$$

where $v$ is the electron velocity, $D_{\varepsilon}$ is the energy diffusion coefficient describing electron heating, $D_{e e(n)}$ and $V_{e e(n)}$ are the coefficients of diffusion and dynamic friction caused by electron-electron (neutral) collisions, and I represents inelastic collision including ionization and excitation for $\mathrm{Ar}$ discharges.

By solving Eqs. (4)-(7), $T_{e}$ could be calculated, and the results are indicated in Fig. 4. In the calculation, the measured gas temperature was used, and the effect of variation of $T_{e}$ by adding the gas temperature on the kinetic model was compared. As not shown here, the calculated EEPFs were in excellent agreement with the measured result: the EEPF shows Maxwellian distribution with a slightly depleted high energy part, and the depleted tail is replenished with the $\mathrm{RF}$ power. If we only consider stepwise ionization in the global model, $T_{e}$ should decrease with an increase in the

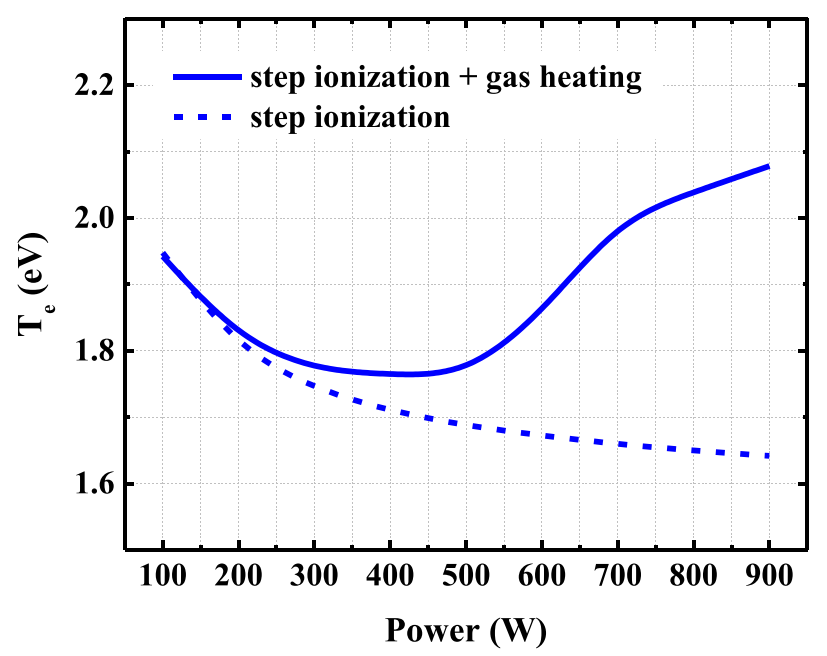

FIG. 4. Calculated electron temperature using the kinetic model with and without gas heating. input power due to the reduction in $\varepsilon_{c}$ by the stepwise ionization, which is represented by dotted lines in Fig. 4. When gas heating is included in the stepwise ionization global model, however, the $T_{e}$ behavior changes abnormally (solid line in Fig. 4). As observed from the results of the kinetic model considering gas heating, $T_{e}$ decreases with low $\mathrm{RF}$ power at first because of the stepwise ionization. After that, $T_{e}$ is saturated with an RF power of approximately 300-500 W, which implies that the effects of the stepwise ionization and the gas heating are balanced in the variation in $T_{e}$. With a further increase in the RF power, $T_{e}$ is remarkably increased, and this indicates that the mechanism for the variation of $T_{e}$ transits from being stepwise ionization-dominated to gas heating-dominated. Therefore, the observed evolution of the $T_{e}$ is mainly due to the transition of the discharge characteristics owing to the contrasting effects of the stepwise ionization and gas heating. The calculated result (solid line in Fig. 4) using the kinetic model including both the stepwise ionization and gas heating is in good agreement with the experimental result shown in Fig. 2(a).

In this letter, we observed the abnormal behavior of $T_{e}$ in ICP. In the low RF power or plasma density region, $T_{e}$ decreased, while it remarkably increased in the high RF power region. It was also demonstrated from the laser Rayleigh scattering measurement that $T_{g}$ slightly increased with low RF powers, and it significantly increased in the high RF power region. The kinetic model, which considers stepwise ionization and gas heating, was developed to analyze the change in $T_{e}$. From the kinetic model analysis, the apparently abnormal trend in $T_{e}$ can be understood by the contrasting effects of stepwise ionization and gas heating. It should be noted that the original notion was that $T_{e}$ is decoupled (or weakly coupled) to the plasma power or plasma density, and thus, $T_{e}$ must remain constant (or slightly decrease) with plasma density in the conventional global model. However, our experiments and improved modeling show that $T_{e}$ has a much stronger relationship with plasma power than we initially expected, and the gas heating effect should be considered.

The authors thank anonymous reviewers. This research was supported by Korea Research Institute of Standard and Science (KRISS) and the R\&D Convergence Program (CAP16-04-KRISS) of National Research Council of Science and Technology (NST) of Republic of Korea, and also supported by R\&D Program of "Plasma BigData ICT Convergence Technology Research Project" through the National Fusion Research Institute of Korea (NFRI) funded by the Government funds.

${ }^{1}$ M. A. Lieberman and A. J. Lichtenberg, Principle of Plasma Discharges and Materials Processing (Wiley, New York, 2005).

${ }^{2} \mathrm{~F}$. Chen, Introduction to Plasma Physics and Controlled Fusion: I. Plasma Physics, 2nd ed. (Plenum, New York, 1984).

${ }^{3}$ P. Chabert and N. Braithwaite, Physics of Radiofrequency Plasmas (Cambridge University Press, Cambridge, 2011).

${ }^{4}$ H.-C. Lee and C.-W. Chung, Plasma Sources Sci. Technol. 23, 062002 (2014).

${ }^{5}$ K. Takahashi, C. Charles, R. W. Boswell, and T. Fujiwara, Phys. Rev. Lett. 107, 035002 (2011).

${ }^{6}$ V. A. Godyak and R. B. Piejak, Appl. Phys. Lett. 63, 3137 (1993).

${ }^{7}$ M. J. Hartig and M. J. Kushner, J. Appl. Phys. 73, 1080 (1993). 
${ }^{8}$ J. Schulze, T. Gans, D. O'Connell, U. Czarnetzki1, A. R. Ellingboe, and M. M. Turner, J. Phys. D: Appl. Phys. 40, 7008 (2007).

${ }^{9}$ H.-C. Lee, M.-H. Lee, and C.-W. Chung, Appl. Phys. Lett. 96, 041503 (2010).

${ }^{10}$ S. Park, W. Choe, S. Y. Moon, and J. Park, Appl. Phys. Lett. 104, 084103 (2014).

${ }^{11}$ V. A. Godyak and R. B. Piejak, Phys. Rev. Lett. 65, 996 (1990).

${ }^{12}$ M. M. Turner, D. A. W. Hutchinson, R. A. Doyle, and M. B. Hopkins, Phys. Rev. Lett. 76, 2069 (1996).

${ }^{13}$ H.-C. Lee and C.-W. Chung, Appl. Phys. Lett. 101, 244104 (2012).

${ }^{14}$ C. Lee and M. A. Lieberman, J. Vac. Sci. Technol., A 13, 368 (1995).

${ }^{15}$ P. N. Wainman, M. A. Lieberman, A. J. Lichtenberg, R. A. Stewart, and C. Lee, J. Vac. Sci. Technol., A 13, 2464 (1995).

${ }^{16}$ M.-H. Lee and C.-W. Chung, Appl. Phys. Lett. 87, 131502 (2005).

${ }^{17}$ V. A. Godyak, R. B. Piejak, and B. M. Alexandrovich, Plasma Sources Sci. Technol. 11, 525 (2002).

${ }^{18}$ J. T. Gudmundsson, Plasma Sources Sci. Technol. 10, 76 (2001).

${ }^{19}$ H.-C. Lee and C.-W. Chung, Sci. Rep. 5, 15254 (2015).

${ }^{20}$ H.-C. Lee and C.-W. Chung, Phys. Plasmas 19, 033514 (2012).

${ }^{21}$ H.-C. Lee and C.-W. Chung, Phys. Plasmas 20, 101607 (2013).

${ }^{22}$ V. A. Godyak and V. I. Demidov, J. Phys. D: Appl. Phys. 44, 269501 (2011).

${ }^{23}$ R. Boyd, Proc. R. Soc., A 201, 329 (1950).

${ }^{24}$ R. Boyd and N. Twiddy, Proc. R. Soc., A 250, 53 (1959).

${ }^{25}$ R. H. Sloane and E. I. R. MacGregor, Philos. Mag. 18, 193 (1934).

${ }^{26}$ S. H. Seo, C. W. Chung, J. I. Hong, and H. Y. Chang, Phys. Rev. E 62, 7155 (2000).

${ }^{27}$ H.-C. Lee, H.-J. Hwang, Y.-C. Kim, J. Y. Kim, D.-H. Kim, and C.-W. Chung, Phys. Plasmas 20, 033504 (2013).

${ }^{28}$ M. J. Druyvesteyn, Z. Phys. A. Hadrons and Nuclei 64, 781 (1930).

${ }^{29}$ D. Kim, H.-C. Lee, Y. Kim, and C. Chung, Appl. Phys. Lett. 103, 084103 (2013).

${ }^{30}$ V. A. Godyak, R. B. Piejak, and B. M. Alexandrovich, Phys. Rev. Lett. 68, 40 (1992).
${ }^{31}$ O. V. Polomarova, C. E. Theodosiou, and I. D. Kaganovich, Phys. Plasmas 12, 080704 (2005).

${ }^{32}$ I. V. Schweigert, Phys. Rev. Lett. 92, 155001 (2004).

${ }^{33}$ E. Schüngel, S. Brandt, Z. Donkó, I. Korolov, A. Derzsi, and J. Schulze, Plasma Sources Sci. Technol. 24, 044009 (2015).

${ }^{34}$ J. Schulze, E. Schüngel, Z. Donkó, and U. Czarnetzki, Plasma Sources Sci. Technol. 20, 015017 (2011).

${ }^{35}$ H.-C. Lee and C.-W. Chung, Plasma Sources Sci. Technol. 24, 024001 (2015).

${ }^{36}$ A. V. Khrabrov, I. D. Kaganovich, P. L. G. Ventzek, A. Ranjan, and L. Chen, Plasma Sources Sci. Technol. 24, 054003 (2015).

${ }^{37}$ Y.-X. Liu, Q.-Z. Zhang, W. Jiang, L.-J. Hou, X.-Z. Jiang, W.-Q. Lu, and Y.-N. Wang, Phys. Rev. Lett. 107, 055002 (2011).

${ }^{38}$ H.-C. Lee, J.-K. Lee, and C.-W. Chung, Phys. Plasmas 17, 033506 (2010).

${ }^{39}$ V. A. Godyak and B. M. Alexandrovich, J. Appl. Phys. 118, 233302 (2015).

${ }^{40}$ B. H. Seo, S. J. You, and J. H. Kim, Jpn. J. Appl. Phys., Part 1 54, 086102 (2015).

${ }^{41}$ B. H. Seo, D. W. Kim, J. H. Kim, and S. J. You, Phys. Plasmas 22, 093510 (2015).

${ }^{42}$ V. M. Donnelly and M. V. Malyshev, Appl. Phys. Lett. 77, 2467 (2000).

${ }^{43}$ G. A. Hebner, J. Appl. Phys. 80, 2624 (1996).

${ }^{44}$ G. Cunge, D. Vempaire, and N. Sadeghi, Appl. Phys. Lett. 96, 131501 (2010).

${ }^{45}$ S. S. Kim, C. W. Chung, and H. Y. Chang, Thin Solid Films 435, 72 (2003).

${ }^{46}$ S. Ashida, C. Lee, and M. A. Lieberman, J. Vac. Sci. Technol., A 13, 2498 (1995).

${ }^{47}$ F. Kannari, M. Obaraa, and T. Fujioka, J. Appl. Phys. 57, 4309 (1985).

${ }^{48}$ N. L. Bassett and D. J. Economou, J. Appl. Phys. 75, 1931 (1994).

${ }^{49}$ D. R. Lide, CRC Handbook of Chemistry and Physics, 80th ed. (CRC Press, Boca Raton, 2000).

${ }^{50}$ M. Schulze, A. Yanguas-Gill, A. Keudell, and P. Awakowicz, J. Phys. D: Appl. Phys. 41, 065206 (2008).

${ }^{51}$ N. Kang, S. Oh, and A. Ricard, J. Phys. D: Appl. Phys. 41, 155203 (2008). 